\title{
COMPACT ACTIONS COMMUTING WITH ERGODIC ACTIONS AND APPLICATIONS TO CROSSED PRODUCTS
}

\author{
C. PELIGRAD
}

\begin{abstract}
Let $(A, K, \beta)$ be a $C^{*}$-dynamical system with $K$ compact. In this paper we prove a duality result for saturated actions (Theorem 3.3). The proof of this result can also be considered as an alternate proof of the corresponding result for von Neumann algebras due to Araki, Haag, Kastler and Takesaki [14]. We also obtain results concerning the simplicity and the primeness of the crossed product $A \times{ }_{\beta} K$ in terms of the ergodicity of the commutant of $\beta$ (Propositions 5.3 and 5.4).
\end{abstract}

\section{INTRODUCTION}

Let $(A, G, \alpha)$ be a $C^{*}$-dynamical system. In [8] the notion of topological transitivity was introduced. Namely $\alpha$ is called topologically transitive if for every two nonzero, globally $G$-invariant, hereditary $C^{*}$-subalgebras $B_{1}, B_{2}$ of $A$, their product is nonzero. As an application of our techniques and notions we proved [8] some duality results for compact abelian actions commuting with ergodic actions. Subsequently in [2] a seemingly stronger notion of ergodicity called strong topological transitivity was considered. The $C^{*}$-dynamical system $(A, G, \alpha)$ is said to be strongly topologically transitive if for every finite sequence $\left\{\left(x_{i}, y_{i}\right) \mid i=1,2, \ldots, n\right\}$ of pairs of elements $x_{i}, y_{i} \in A$ for which $\sum x_{i} \otimes y_{i} \neq 0$ in the algebraic tensor product $A \otimes A$, there is an $g_{0} \in G$ such that $\sum_{i=1}^{n} x_{i} \alpha_{g_{0}}\left(y_{i}\right) \neq 0$ in $A$. It is easy to see that this condition for $n=1$ is equivalent with topological transitivity. Using this stronger notion, in [2] a duality result for compact, not necessarily abelian actions is proved. We will call an action $\alpha$ weakly ergodic if the fixed point algebra $M(A)^{\alpha}$ is trivial (where $M(A)$ denotes the multiplier algebra of $A$ ). In this paper (Theorem 3.3), we prove a duality result for compact actions which are saturated in the sense of Rieffel. The proof of Theorem 3.3 can also be viewed as an alternate proof of the corresponding result for von Neumann algebras due to Araki, Haag, Kastler and Takesaki [14]. We also obtain a characterization of the generators of the one-parameter subgroups of a saturated compact group action in the presence of an ergodic commuting action (Theorem 4.1).

Finally in $\S 5$ we consider the problem of deciding when a cross product $C^{*}$-algebra by a compact action is simple or prime. Using our earlier results contained in [10] we obtain results concerning the simplicity and primeness of

Received by the editors December 3, 1987 and, in revised form, March 23, 1990.

1980 Mathematics Subject Classification (1985 Revision). Primary 46L55; Secondary 43A45.

Supported in part by NSF Grant No. DMS-8502353. 
the cross product in terms of the ergodicity of the commutant of the (compact) action. We then indicate some open problems in this theory.

I am indebted to the referee for finding several mistakes in the initial version of this paper.

\section{PRELIMINARY NOTIONS AND RESUltS}

Let $K$ be a compact group. We shall denote by $\widehat{K}$ the dual of $K$ (i.e. the set of all unitary equivalence classes of irreducible representations of $K$ ). For each $\pi \in K$ we also denote by $\pi$ a representative of the class $\pi$ and let $\left[\pi_{i j}(k)\right]$ be the matrix of $\pi_{k}$ in $B\left(H_{\pi}\right)$ where $H_{\pi}$ is the finite dimensional Hilbert space on which $\pi$ acts. Let also $\chi_{\pi}(k)=(\operatorname{dim} \pi)\left(\operatorname{tr}\left(\pi_{k^{-1}}\right)\right.$ be the normalized character of $\pi$.

Let $(A, k, \beta)$ be a $C^{*}$-dynamical system with $K$ compact. For every $\pi \in \widehat{K}$ we consider the following mapping $P_{\pi}^{\beta}: A \rightarrow A$,

$$
P_{\pi}^{\beta}(a)=\int_{K} \overline{\chi_{\pi}(k)} \beta_{k}(a) d k .
$$

We also denote the following map from $A$ to $A$ by $P_{\pi, i, j}$,

$$
P_{\pi, i, j}^{\beta}(a)=\int_{K} \overline{\pi_{j i}(k)} \beta_{k}(a) d k .
$$

We remark, for further use, the following relations

$$
\begin{gathered}
\text { (a) } P_{\pi}^{\beta} P_{\pi, i, j}^{\beta}=P_{\pi, i, j}^{\beta} P_{\pi}^{\beta}=P_{\pi, i, j}^{\beta}, \quad \text { (b) } P_{\pi, i, j}^{\beta} P_{\pi, k, l}^{\beta}=\delta_{i l} P_{\pi, k, j}^{\beta} . \\
\beta_{k}\left(P_{\pi, i, j}^{\beta}(a)\right)=\sum_{l} \pi_{l j}(k) P_{\pi, i, l}^{\beta}(a), \quad k \in K .
\end{gathered}
$$

If we denote the matrix in $B \otimes B\left(H_{\pi}\right)$ with entries $P_{\pi, i, j}^{\beta}(a)$ by $\left[P_{\pi, i, j}^{\beta}(a)\right]$, then (4) is equivalent with

$$
\left(\beta_{k} \otimes 1\right)\left(\left[P_{\pi, i, j}^{\beta}(a)\right]\right)=\left[P_{\pi, i, j}^{\beta}(a)\right]\left(1 \otimes \pi_{k}\right), \quad k \in K .
$$

We make the following notations:

$$
A_{1}^{\beta}(\pi)=\left\{a \in A \mid P_{\pi}^{\beta}(a)=a\right\}
$$

and

$$
A_{2}^{\beta}(\pi)=\left\{\left[a_{i j}\right] \in A \otimes B\left(H_{\pi}\right) \mid\left(\beta_{k} \otimes i\right)\left(\left[a_{i j}\right]\right)=\left[a_{i j}\right]\left(1 \otimes \pi_{k}\right)\right\} .
$$

If $\pi=i$, the trivial one dimensional representation, we use the notation $A_{1}^{\beta}(i)=A^{\beta}$ for the fixed point algebra of the action.

Clearly $A_{2}^{\beta}(\pi) A_{2}^{\beta}(\pi)^{*} \subset A \otimes B\left(H_{\pi}\right)^{\beta \otimes i}$ and $A_{2}^{\beta}(\pi)^{*} A_{2}^{\beta}(\pi) \subset A \otimes B\left(H_{\pi}\right)^{\beta \otimes a d \pi}$ are two-sided ideals for every $\pi \in \widehat{K}$. By [6] we have the inclusion

$$
\left\{\left[P_{\pi, i, j}^{\beta}(a)\right] \mid a \in A\right\} \subset A_{2}^{\beta}(\pi) .
$$

Conversely, if $\left[a_{i j}\right] \in A_{2}(\pi)$, then by denoting $a=\sum a_{i i}$, a standard calculation using the orthogonality relations shows that $P_{\pi, i, j}(a)=a_{i j}$ for all $1 \leq i, j$ $\leq \operatorname{dim} \pi$ so that we have the following equality

$$
\left\{\left[P_{\pi, i, j}^{\beta}(a)\right] \mid a \in A\right\}=A_{2}^{\beta}(\pi) .
$$


As remarked by Rieffel (see [11, Chapter 7]), $A$ has a natural structure of $A^{\beta}-A \times{ }_{\beta} K$-bimodule. If $A$ with this structure is an imprimitivity bimodule (see [12]) for the definition of imprimitivity bimodules) then the action is called saturated. In this case $A^{\beta}$ and $A \times_{\beta} K$ are strongly Morita equivalent in the sense of Rieffel [12]. In [10] it is proved that the action $\beta$ is saturated if and only if $\overline{A_{2}^{\beta}(\pi)^{*} A_{2}^{\beta}(\pi)}=A \otimes B\left(H_{\pi}\right)^{\alpha \otimes \text { ad } \pi}$ for every $\pi \in \widehat{K}$.

In [10] we have obtained necessary and sufficient conditions in order that the crossed product $A \times_{\beta} K$ of $A$ by a compact group action $(K, \beta)$, be simple or prime. Namely, we have proved, in particular, the following result:

Theorem A. Let $(A, K, \beta)$ be a $C^{*}$-dynamical system with $K$ compact. Then the following two conditions are equivalent:

(1) $A \times_{\beta} K$ is simple (respectively prime),

(2) (a) The fixed point algebras $A \otimes B\left(H_{\pi}\right)^{\beta \otimes \mathrm{ad} \pi}$ are simple (respectively prime) for every $\pi \in \widehat{K}$,

(b) $A_{1}^{\beta}(\pi) \neq 0$ for every $\pi \in \widehat{K}$.

For the trivial one dimensional representation $\pi$ we obviously have $A \otimes$ $B\left(H_{\pi}\right)^{\beta \otimes \mathrm{ad} \pi}=A^{\beta}$. We have shown in [10, Example 3.9] that the simplicity (resp. primeness) of $A^{\beta}$ together with the condition (2)(b) does not necessarily imply the simplicity (resp. primeness) of the crossed product $A \times{ }_{\beta} K$.

\section{DuAlity FOR COMPACT ACTIONS}

Let $(A, K, \beta)$ be a $C^{*}$-dynamical system with $K$ compact. We denote by $\operatorname{Aut}_{\beta}(A)$ the group of all ${ }^{*}$ automorphisms of $A$ that commute with $\beta_{k}$ for every $k \in K$.

3.1 Remark. If $\rho \in$ Aut $\beta(A)$ and $\pi \in \widehat{K}$ then $\rho\left(A_{1}^{\beta}(\pi)\right) \subset A_{1}^{\beta}(\pi)$ and $\rho\left(A_{2}^{\beta}(\pi)\right)$ $\subset A_{2}^{\beta}(\pi)$. The proof of these inclusions is straightforward.

3.2 Remark. An easy adaptation of [4, Theorem 2.1] shows that there exists an approximate identity $\left(e_{\lambda}\right)_{\lambda \in \Lambda}$ of $A_{2}^{\beta}(\pi)^{*} A_{2}^{\beta}(\pi)$ of the form $e_{\lambda}=\sum_{i=1}^{n_{\lambda}}\left(a_{i}^{\lambda}\right)^{*} a_{i}^{\lambda}$ where $a_{i}^{\lambda} \in A_{2}^{\beta}(\pi)$.

Let $\Pi(K)$ be the category of all finite dimensional unitary representations of $K$. In addition to the usual categorical operations, $\Pi(K)$ admits the operation of tensor multiplication of any two objects. It also admits the operation of involution which associates with the object $\pi \in \mathrm{Ob} \Pi(K)$ the adjoint representation $\pi^{*}$.

We shall consider the category $\Pi(K)$ as the dual object of the group $K$. In general $\Pi(K)$ is obtained from the set $\widehat{K}$ by specifying the involution * : $\pi \rightarrow \pi^{*}$ on $\widehat{K}$ and for every $\pi_{1}, \pi_{2} \in \widehat{K}$ the isomorphism of the space $H_{\pi_{1}} \otimes H_{\pi_{2}}$ $\left(\approx H_{\pi_{1} \otimes \pi_{2}}\right)$ onto a direct sum of the form $\sum_{\pi \in \widehat{K}} n_{\pi_{1}, \pi_{2}}^{\pi} H_{\pi}$. Here, $H_{\pi}$ denotes the space of the representation $\pi$ and $n_{\pi_{1}, \pi_{2}}^{\pi}$ denotes the multiplicity of the irreducible representation $\pi$ in $\pi_{1} \otimes \pi_{2}$.

By a representation of the category $\Pi(K)$ we shall mean a nonzero function $\varphi$ on $\mathrm{Ob} \Pi(K)$ taking values in $B\left(H_{\pi}\right)$ at the point $\pi \in \mathrm{Ob} \Pi(K)$ and satisfying the following conditions:

(i) $S \varphi\left(\pi_{1}\right)=\varphi\left(\pi_{2}\right) S$ for every operator $S$ that intertwines $\pi_{1}$ and $\pi_{2}$.

(ii) $\varphi\left(\pi_{1} \otimes \pi_{2}\right)=\varphi\left(\pi_{1}\right) \otimes \varphi\left(\pi_{2}\right)$. 
We denote by $\Gamma(\Pi(K))$ the set of all representations of $\Pi(K)$. Then $\Gamma(\Pi(K))$ has a natural operation and a natural topology under which it is a compact topological group. Tannaka's duality theorem [5] asserts that $K$ and $\Gamma(\Pi(K))$ are isomorphic as topological groups under the isomorphism $k \rightarrow \varphi_{k}$ where $\varphi_{k}(\pi)=\pi_{k}, \pi \in \mathrm{Ob} \Pi(K), k \in K$.

We can now state and prove our main result of this section.

3.3 Theorem. Let $(A, K, \beta)$ be a $C^{*}$-dynamical system with $K$ compact and $\beta$ saturated. Assume that there exists a weakly ergodic action $(G, \alpha)$ on $A$ such that $\alpha_{G} \subset \operatorname{Aut}_{\beta}(A)$.

If $\sigma \in \operatorname{Aut}(A)$ leaves $A^{\beta}$ pointwise invariant and commutes with $\alpha_{G}$ then $\sigma=\beta_{k}$ for some $k \in K$.

Proof. Let $\pi \in \widehat{K}$. Since $\beta$ is saturated we have by [10, Theorem 3.3] that $\overline{A_{2}^{\beta}(\pi)^{*} A_{2}^{\beta}(\pi)}=A \otimes B\left(H_{\pi}\right)^{\alpha \otimes \mathrm{ad} \pi}$. Let $e_{\lambda}=\sum_{i=1}^{n_{\lambda}} a_{i}^{\lambda *} a_{i}^{\lambda}$ be an approximate identity as in Remark 3.2. Then (strong) $\lim _{\lambda \in \Lambda} e_{\lambda}=1 \otimes 1 \in M(A) \otimes B\left(H_{\pi}\right)$.

Let now $\sigma \in \operatorname{Aut}(A)$ be such that $\sigma \alpha_{g}=\alpha_{g} \sigma$ for every $g \in G$ and $\left.\sigma\right|_{A} \beta=$ id. Denote by $\tilde{\sigma}=\sigma \otimes i$ the extension of $\sigma$ to $A \otimes B(H \pi)$ and let $a \in A_{2}^{\beta}(\pi)$ be an arbitrary element. We have

$$
\tilde{\sigma}\left(a e_{\lambda}\right)=\sum_{i=1}^{n_{\lambda}} \tilde{\sigma}\left(a a_{i}^{\lambda *} a_{i}^{\lambda}\right)=\sum a a_{i}^{\lambda *} \tilde{\sigma}\left(a_{i}^{\lambda}\right)=a z_{\lambda}
$$

where

$$
z_{\lambda}=\sum_{i=1}^{n_{\lambda}} a_{i}^{\lambda *} \tilde{\sigma}\left(a_{i}^{\lambda}\right)
$$

Let $B=A \otimes B\left(H_{\pi}\right) \otimes B\left(C^{n_{\lambda}}\right)$. If we denote

$$
a^{\lambda}=\left[\begin{array}{cccc}
a_{1}^{\lambda} & 0 & \ldots & 0 \\
a_{2}^{\lambda} & 0 & \ldots & 0 \\
\vdots & & & \\
a_{n_{\lambda}} & 0 & \ldots & 0
\end{array}\right] \in B
$$

we have

$$
\left(a^{\lambda}\right)^{*}\left(\tilde{\sigma} \otimes i_{n_{\lambda}}\right)\left(a^{\lambda}\right)=\left[\begin{array}{cccc}
z_{\lambda} & 0 & \ldots & 0 \\
\vdots & & & 0 \\
0 & 0 & \ldots & 0
\end{array}\right] .
$$

Therefore $\left\|z_{\lambda}\right\| \leq\left\|a^{\lambda}\right\|^{2}=\left\|e_{\lambda}\right\| \leq 1$. On the other hand if we fix $\lambda_{0} \in \Lambda$ we have

$$
\begin{aligned}
e_{\lambda_{0}} z_{\lambda} & =\sum_{i, j=1}^{n_{\lambda}}\left(a_{i}^{\lambda_{0}}\right)^{*} a_{i}^{\lambda_{0}}\left(a_{j}^{\lambda}\right)^{*} \sigma\left(a_{j}^{\lambda}\right)=\sum_{i, j}\left(a_{i}^{\lambda_{0}}\right)^{*} \tilde{\sigma}\left(a_{i}^{\lambda_{0}}\left(a_{j}^{\lambda}\right)^{*} a_{j}^{\lambda}\right) \\
& =\sum_{i}\left(a_{i}^{\lambda_{0}}\right)^{*} \tilde{\sigma}\left(a_{i}^{\lambda_{0}} \sum_{j}\left(a_{j}^{\lambda}\right)^{*} a_{j}^{\lambda}\right)=\sum_{i}\left(a_{i}^{\lambda_{0}}\right)^{*} \tilde{\sigma}\left(a_{i}^{\lambda_{0}} e_{\lambda}\right) .
\end{aligned}
$$

Hence (norm) $\lim _{\lambda \in \Lambda} e_{\lambda_{0}} z_{\lambda}=z_{\lambda_{0}}$. Since $\left(e_{\lambda}\right)$ is an approximate identity of $\left(A \otimes B\left(H_{\pi}\right)\right)^{\alpha \otimes \mathrm{ad} \pi}$ it is immediate to show that it is an approximate identity 
of $A \otimes B\left(H_{\pi}\right)$. From these one can infer that there exists an element $z_{\pi} \in$ $M(A) \otimes B\left(H_{\pi}\right)$ such that:

(1) (norm) $\lim _{\lambda \in \Lambda} a z_{\lambda}=a z_{\pi}, a \in A$, and

(2) $\tilde{\sigma}(a)=a z_{\pi}$ for every $a \in A_{2}^{\beta}(\pi)$. Since by hypothesis $\left[\sigma, \alpha_{g}\right]=0$, $g \in G$, we obviously have $\left[\tilde{\sigma}, \tilde{\alpha}_{g}\right]=0$ for every $g \in G$. Therefore, from (2) we infer

(3) $\tilde{\alpha}_{g}(\tilde{\sigma}(a))=\tilde{\alpha}_{g}(a) \tilde{\alpha}_{g}\left(z_{\pi}\right), g \in G$. On the other hand, using Remark 3.1 we have

(4) $\tilde{\alpha}_{g}(\sigma(\tilde{a}))=\tilde{\sigma}\left(\tilde{\alpha}_{g}(a)\right)=\tilde{\alpha}_{g}(a) z_{\pi}, g \in G$, and hence

(5) $\tilde{\alpha}_{g}(a) \tilde{\alpha}_{g}\left(z_{\pi}\right)=\tilde{\alpha}_{g}(a) z_{\pi}, g \in G, a \in A_{2}^{\beta}(\pi)$.

Using (5) for $a=a_{i}^{\lambda}$ one can see that $\tilde{\alpha}_{g}\left(z_{\pi}\right)=z_{\pi}, g \in G$. Since, by assumption $G$ acts weakly ergodically on $A$, it follows that $z_{\pi} \in 1 \otimes B\left(H_{\pi}\right)$ for every $\pi \in \widehat{K}$. Let now $\rho$ be an arbitrary finite dimensional representation of $K$. Then $\rho$ can be written as a direct sum of irreducible representations $\rho=\bigoplus \pi_{i}$. By definition put

$$
A_{2}^{\beta}(\rho)=\bigoplus_{i=1}^{n} A_{2}^{\beta}\left(\pi_{i}\right) \quad \text { and } \quad z_{\rho}=\bigoplus_{i=1}^{n} z_{\pi_{i}} .
$$

We have therefore associated to each finite dimensional representation $\rho$ of $K$ an operator $z_{p} \in B\left(H_{\rho}\right)$ where $H_{\rho}$ is the Hilbert space of $\operatorname{dimension} \operatorname{dim} \rho$. We shall prove that $\rho \rightarrow z_{\rho}$ is a representation of the category $\Pi(K)$ in the sense discussed above. Let $S$ be an operator that intertwines $\rho_{1}$ and $\rho_{2}$, namely $S: H_{\rho_{1}} \rightarrow H_{\rho_{2}}$ is such that $S \rho_{1}=\rho_{2} S$. We shall prove that $S z_{\rho_{1}}=$ $z_{\rho_{2}} S$. It is enough to consider the case when one of the two representations, say $\rho_{1}$, is irreducible. If $\rho_{1}$ does not occur in $\rho_{2}$ then the only intertwining operator is $S=0$ and in this case the equality we have to prove is obvious. Assume now that $\rho_{1}$ does occur in $\rho_{2}$ with multiplicity $m$. Then

$$
\rho_{2}=\underbrace{\rho_{1} \oplus \rho_{1} \oplus \cdots \oplus \rho_{1}}_{m} \oplus \rho_{3}
$$

and $\rho_{1}$ does not occur in $\rho_{3}$. Let $S$ be the following intertwining operator

$$
S x=0 \oplus \cdots \oplus 0 \oplus \times_{j} \oplus 0 \oplus \cdots \oplus 0, \quad j \leq m, x \in H_{\rho_{1}} .
$$

Since by definition $z_{\rho_{2}}=m z_{\rho_{1}} \oplus z_{\rho_{3}}$, we obviously have $S z_{\rho_{1}}=z_{\rho_{2}} S$. Since every operator that intertwines $\rho_{1}$ and $\rho_{2}$ is a linear combination of operators of the above form we are done. We now prove that $z_{\rho_{1}} \otimes \rho_{2}=z_{\rho_{1}} \otimes z_{\rho_{2}}$.

Let $a=\left[a_{i j}\right] \in A_{2}^{\beta}\left(\pi_{1}\right)$ and $b=\left[b_{i j}\right] \in A_{2}^{\beta}\left(\pi_{2}\right)$. Consider the following matrix

$$
a \odot b=\left[a\left[\begin{array}{cccc}
b_{i j} & 0 & \ldots & 0 \\
0 & b_{i j} & \ldots & 0 \\
\cdot & \cdot & & \\
\cdot & \cdot & & \\
0 & 0 & \ldots & b_{i j}
\end{array}\right]\right] .
$$

The $(i j)$ entry of $a \odot b$ equals

$$
a\left[\begin{array}{cccc}
b_{i j} & 0 & \ldots & 0 \\
0 & b_{i j} & \ldots & 0 \\
\cdot & \cdot & & \\
\cdot & \cdot & & \\
0 & 0 & \ldots & b_{i j}
\end{array}\right]
$$


where

$$
\left[\begin{array}{cccc}
b_{i j} & 0 & \ldots & 0 \\
0 & b_{i j} & \ldots & 0 \\
\cdot & \cdot & & \\
\cdot & \cdot & & \\
0 & 0 & \ldots & b_{i j}
\end{array}\right]
$$

is a $\operatorname{dim} \rho_{1} \times \operatorname{dim} \rho_{1}$ diagonal matrix with all the diagonal elements equal to $b_{i j}$.

Claim 1. $a \odot b \in A_{2}^{\beta}\left(\pi_{1} \otimes \pi_{2}\right)$.

Proof of Claim 1. We have

$$
\begin{aligned}
& \tilde{\beta}_{k}(a \odot b)=\left[\tilde{\beta}_{k} a\left[\begin{array}{cccc}
b_{i j} & 0 & \ldots & 0 \\
0 & b_{i j} & \ldots & 0 \\
\cdot & \cdot & & \\
\cdot & \cdot & & \\
0 & 0 & \ldots & b_{i j}
\end{array}\right]\right] \\
& =\left[\tilde{\beta}_{k}(a)\left[\begin{array}{cccc}
\beta_{k}\left(b_{i j}\right) & 0 & \ldots & 0 \\
0 & \beta_{k}\left(b_{i j}\right) & \ldots & 0 \\
\cdot & \cdot & & \cdot \\
\cdot & \cdot & & \dot{\beta_{k}} \\
0 & 0 & \ldots & \left.\beta_{i j}\right)
\end{array}\right]\right] \\
& =\left[a\left(\rho_{1}\right)_{k}\left[\begin{array}{cccc}
\beta_{k}\left(b_{i j}\right) & 0 & \ldots & 0 \\
0 & \beta_{k}\left(b_{i j}\right) & \ldots & 0 \\
\cdot & \cdot & \cdot & \\
\cdot & \cdot & \cdot & \\
0 & 0 & \ldots & \beta_{k}\left(b_{i j}\right)
\end{array}\right]\right] \\
& =\left[a\left[\begin{array}{cccc}
\beta_{k}\left(b_{i j}\right) & 0 & \ldots & 0 \\
0 & \beta_{k}\left(b_{i j}\right) & \ldots & 0 \\
\cdot & \cdot & & \cdot \\
\cdot & \cdot & & \cdot \\
0 & 0 & \ldots & \beta_{k}\left(b_{i j}\right)
\end{array}\right]\left(\rho_{1}\right)_{k}\right] \text {. }
\end{aligned}
$$

Since $b=\left[b_{i j}\right] \in A_{2}^{\beta}\left(\rho_{2}\right)$ we have $\widetilde{\beta}_{k}(b)=b\left(\rho_{2}\right)_{k}$ and therefore $\beta_{k}\left(b_{i j}\right)=$ $\sum_{l} b_{i l} \rho_{2}^{l j}(k)$. It follows that $\widetilde{\beta}_{k}(a \odot b)$ is the matrix whose $(i j)$ entry is the $\operatorname{dim} \rho_{1} \times \operatorname{dim} \rho_{1}$ matrix

$$
a\left[\begin{array}{cccc}
\left(b\left(\rho_{2}\right)_{k}\right)_{i j} & 0 & \ldots & 0 \\
\cdot & & & \cdot \\
\cdot & & & \dot{\cdot} \\
\dot{0} & \ldots & & \left(b\left(\rho_{2}\right)_{k}\right)_{i j}
\end{array}\right]\left(\rho_{1}\right)_{k} .
$$

An inspection shows that this last matrix equals $(a \odot b)\left(\left(\rho_{1}\right)_{k} \otimes\left(\rho_{2}\right)_{k}\right)$. Therefore, we have

(6) $\widetilde{\beta}_{k}(a \odot b)=(a \odot b)\left(\left(\rho_{1}\right)_{k} \otimes\left(\rho_{2}\right)_{k}, k \in K\right.$. From (6) it follows that $a \odot b \in A_{2}^{\beta}\left(\rho_{1} \otimes \rho_{2}\right)$. The above discussion implies

(7) $\tilde{\sigma}(a \odot b)=(a \odot b) z_{\rho_{1}} \otimes z_{\rho_{2}}$.

Claim 2. $\sigma(a \odot b)=(a \odot b)\left(z_{\rho_{1}} \otimes z_{\rho_{2}}\right)$. 
Proof. The proof of Claim 2 can be done on the same lines as that of the previous claim by taking into account that $\tilde{\sigma}(a)=a z_{\rho_{1}}$ and $\tilde{\sigma}(b)=b z_{\rho_{2}}$.

We can now prove that $z_{\rho_{1}} \otimes_{\rho_{2}}=z_{\rho_{1}} \otimes z_{\rho_{2}}$. Using the relation (7) and Claim 2 we have

(8) $(a \odot b)^{*} \tilde{\sigma}(a \odot b)=c\left(z_{\rho_{1}} \otimes_{\rho_{2}}\right)=c\left(z_{\rho_{1}} \otimes z_{\rho_{2}}\right)$ where $c=\left[c_{i j}\right]$ is defined as follows

$$
c_{i j}=\sum_{k}\left[\begin{array}{cccccc}
b_{k i}^{*} & 0 & \ldots & 0 & & \\
0 & & & & & \\
\cdot & & & 0 & \ldots & b_{k i}^{*}
\end{array}\right] a^{*} a\left[\begin{array}{cccc}
b_{k j} & 0 & \ldots & 0 \\
0 & & & \\
\cdot & & & \\
\cdot & & & \\
0 & 0 & \ldots & b_{k j}
\end{array}\right] .
$$

By Remark 3.2 there is an approximate identity $e_{\lambda}$ of $A \otimes B\left(H_{\rho_{1}}\right)$ of the form $e_{\lambda}=\sum_{i=1}^{n_{\lambda}} a_{i}^{*} a_{i}$ with $a_{i} \in A_{2}^{\beta}\left(\rho_{1}\right)$ and an approximate identity $\left(f_{\nu}\right)$ of $A \otimes$ $B\left(H_{\rho_{2}}\right)$ of the form $f_{\nu}=\sum_{i=1}^{n_{\nu}} b_{i}^{*} b_{i}$ with $b_{i} \in A_{2}^{\beta}\left(\rho_{2}\right)$. Using this in the relation (8) it immediately follows that $z_{\rho_{1} \otimes \rho_{2}}=z_{\rho_{1}} \otimes z_{\rho_{2}}$. Therefore $\rho \rightarrow z_{\rho}$ is a representation of the category $\pi(K)$ in the sense defined above. By Tannaka's duality theorem, there is a $k_{0} \in K$ such that $z_{\rho}=\rho_{k_{0}}$ for every $\rho \in \pi(K)$. It follows that $\tilde{\sigma}(a)=a \pi_{k_{0}}$ for every $\pi \in \widehat{K}$ and $a \in A_{2}^{\beta}(\pi)$. Therefore $\sigma=\beta_{k_{0}}$ and the proof is completed.

\section{ONE-PARAMETER SUBGROUPS OF $\beta_{K}$ AND THEIR GENERATORS}

In this section we shall give an infinitesimal characterization of the oneparameter subgroups of $\beta_{K}$ (where $(K, \beta)$ is the action on $A$ considered in Theorem 3.3).

4.1 Theorem. Let $(A, K, \beta)$ be a $C^{*}$-dynamical system with $K$ compact and $\beta$ saturated. Assume that there exists a weakly ergodic action $(G, \alpha)$ on $A$ such that $\alpha_{G} \operatorname{Aut}_{\beta}(A)$. Let $\delta$ be a symmetric derivation on $A$ with domain $D(\delta)=\sum_{\pi \in \widehat{K}} A_{1}^{\beta}(\pi)$. Then the following conditions are equivalent:

(1) (a) $\delta(x)=0, x \in A^{\beta}$, (b) $\delta\left(\alpha_{g}(x)\right)=\alpha_{g}(\delta(x)), x \in D(\delta), g \in G$, and

(2) $\delta$ is closable and its closure $\bar{\delta}$ generates a one-parameter subgroup of $\beta_{K}$. Proof. Obviously $(2) \rightarrow(1)$. We will prove that $(1) \rightarrow(2)$. We denote by $\tilde{\delta}$ the extension of $\delta$ to $A \otimes B\left(H_{\pi}\right)$ for various $\pi \in \Pi(K)$, given by $\tilde{\delta}=\delta \otimes i$. By [3, Theorem 2.3.8], the restriction of $\delta$ to $A_{1}^{\beta}(\pi)$ is bounded for every $\pi \in \widehat{K}$. Hence the restriction of $\tilde{\delta}$ to $A_{2}^{\beta}(\pi)$ is bounded for every $\pi \in \widehat{K}$. Using arguments similar to those used in the first part of the proof of Theorem 3.3 it can be shown that for every $\pi \in \Pi(K)$ there is an $s_{\pi} \in B\left(H_{\pi}\right)$ such that $\tilde{\delta}(a)=a s_{\pi}$ for all $a \in A_{2}^{\beta}(\pi)$. By the relation (8) in $\S 2$ we have

$$
\delta(x)=\sum_{i, 1} P_{\pi, i, 1}^{\beta}(x)\left(s_{\pi}\right)_{1 i}
$$

for every $x \in A_{1}^{\beta}(\pi)$ and $\pi \in \widehat{K}$. In particular, it follows that $\delta\left(A_{1}^{\beta}(\pi)\right) \subset$ $A_{1}^{\beta}(\pi)$ for every $\pi \in \widehat{K}$. Let now $\left\{a_{n}\right\}_{n=1}^{\infty} \subset \sum_{\pi} A_{1}^{\beta}(\pi)$ be a sequence such that $\lim _{n \rightarrow \infty} a_{n}=0$ and $\lim _{n \rightarrow \infty} \delta\left(a_{n}\right)=b$. Since $a_{n} \in \sum_{\pi} A_{1}^{\beta}(\pi)$, we can write 
$a_{n}=\sum_{j=1}^{k_{n}} P_{\pi_{j} n}^{\beta}\left(a_{n}\right)$. Let $\pi_{0} \in \widehat{K}$. Then we have

$$
\begin{aligned}
P_{\pi_{0}}^{\beta}\left(\delta\left(a_{n}\right)\right) & =\int_{K} \chi_{\pi_{0}}(k) \beta_{k}\left(\delta\left(a_{n}\right)\right) d k \\
& =\sum_{j=1}^{k_{n}} \int_{K} \chi_{\pi_{0}}(k) \beta_{k}\left(\delta\left(P_{\pi_{j}}^{\beta} n\left(a_{n}\right)\right)\right) d k \\
& =\sum_{j=1}^{k_{n}} \int_{K} \chi_{\pi_{0}}(k) \beta_{k}\left(\sum_{i, 1} P_{\pi_{j, i, 1}^{n}}\left(a_{n}\right)\left(s_{\pi_{j}^{n}}\right)_{1 i}\right) d k \\
& =\sum_{i, l} \int_{K} \chi_{\pi_{0}}(k) \beta_{k}\left(P_{\pi_{0, i, l}}^{\beta}\left(a_{n}\right)\right) d k\left(s_{\pi_{0}}\right)_{l i} .
\end{aligned}
$$

Since $\lim _{n \rightarrow \infty} a_{n}=0$, we have that $\lim _{n \rightarrow \infty} P_{\pi_{0} i, 1}^{\beta}\left(a_{n}\right)=0$ for all $\pi_{0} \in \widehat{K}$ and $1 \leq i, 1 \leq \operatorname{dim} \pi_{0}$. Therefore $\lim _{\pi_{0}}^{\beta}\left(\delta\left(a_{n}\right)\right)=0$. But, at the same time $\lim _{n \rightarrow \infty} P_{\pi_{0}}\left(\delta\left(a_{n}\right)\right)=P_{\pi_{0}}^{\beta}(b)$ for every $\pi_{0} \in \widehat{K}$. Therefore, $P_{\pi_{0}}^{\beta}(b)=0$ for every $\pi_{0} \in \widehat{K}$ and hence $b=0$. Hence $\delta$ is closable. On the other hand, just repeating word by word the proof of Theorem 3.3 it can be shown that for each $t \in \mathbf{R}, \pi \rightarrow \exp \left(t s_{\pi}\right), \pi \in \Pi(K)$, represents an element $k_{t} \in K$ and that $\left\{\alpha_{k_{t}}\right\}$, $t \in \mathbf{R}$, is a one-parameter subgroup of $\alpha_{K}$ that has $\bar{\delta}$ as its generator.

\section{COMPACT ACTIONS COMMUTING WITH ERGODIC ACTIONS AND APPLICATIONS TO CROSSED PRODUCTS}

In this section we shall prove that in the presence of an ergodic action commuting with the compact action, the simplicity (primeness) of the fixed point algebra $A^{\beta}$ is equivalent with the simplicity (primeness) of the crossed product $A \times{ }_{\beta} K$. Recall that an action $(G, \alpha)$ on $A$ is called minimal if there are no nontrivial hereditary $C^{*}$-subalgebras of $A$ which are globally $\alpha$-invariant.

5.1 Lemma. Let $(K, \beta),(G, \alpha)$ be two commuting actions on the $C^{*}$-algebra $A$ with $K$ compact and $\alpha$ minimal. Then $A^{\beta}$ is $G$-simple (i.e., $A^{\beta}$ has no nontrivial $\alpha$-invariant two-sided ideals).

Proof. Let $J A^{\beta}$ be a $G$-invariant two-sided ideal, $J \neq(0)$. Then $B=\overline{J A J}$ is a $G$-invariant hereditary $C^{*}$-subalgebra of $A, B \neq(0)$. Since $\alpha$ is minimal we have $B=A$. It is immediately checked that $B^{\beta}=J$. Therefore $J=A^{\beta}$.

5.2 Proposition. Let $(K, \beta)$ and $(G, \alpha)$ be two commuting actions on $A$ with $K$ compact and $\alpha$ minimal. If $\beta$ is faithful then $A_{1}^{\beta}(\pi) \neq(0)$ for every $\pi \in \widehat{K}$.

Proof. By formula (8) in $\S 2 A_{1}^{\beta} \neq(0)$. But $A_{2}^{\beta}(\pi)$ can be defined for every finite dimensional representation $\pi \in \Pi(K)$. Let $P=\left\{\pi \in \Pi(K) \mid A_{2}^{\beta}(\pi) \neq\right.$ $(0)\}$. Denote by $[P]$ the smallest subset of $\Pi(K)$ which has the following two properties:

(a) If $\pi \in[P]$ then $\pi^{*} \in[P]$.

(b) If $\pi_{1}, \pi_{2} \in[P]$ and $\pi_{1} \otimes \pi_{2}=\sum^{\oplus} \pi^{j}, \pi^{j} \in \widehat{K}$, then $\pi^{j} \in[P]$ for all $j$. We shall prove that $P=[P]$. Obviously $P$ satisfies property (a). To prove that $P$ also satisfies (b) it is enough to take $\pi_{1}, \pi_{2} \in P \cap \widehat{K}$. 
As remarked in $\S 2, A_{2}^{\beta}\left(\pi_{1}\right) A_{2}^{\beta}\left(\pi_{1}\right)^{*}$ and $A_{2}^{\beta}\left(\pi_{2}\right) A_{2}^{\beta}\left(\pi_{2}\right)^{*}$ are two-sided ideals of $A^{\beta} \otimes B\left(H_{\pi}\right)$. It is immediate that these ideals are $G$-invariant (because $\alpha$ and $\beta$ commute). Since every such ideal of $A^{\beta} \otimes B\left(H_{\pi}\right)$ is of the form $J \otimes B\left(H_{\pi}\right)$ for some $G$-invariant ideal $J$ of $A^{\beta}$, Lemma 5.1 implies that

$$
\overline{A_{2}^{\beta}\left(\pi_{i}\right) A_{2}^{\beta}\left(\pi_{i}^{*}\right)}=A^{\beta} \otimes B\left(H_{\pi_{i}}\right), \quad i=1,2 .
$$

Let $e_{1}^{\lambda_{1}}=\sum_{i=1}^{n_{\lambda_{1}}} A_{i} A_{i}^{*}, A_{i} \in A_{2}^{\beta}\left(\pi_{1}\right)$, be an approximate identity of $A^{\beta} \otimes B\left(H_{\pi_{2}}\right)$, and similarly $e_{2}^{\lambda_{2}}=\sum_{i=1}^{m_{\lambda_{2}}} B_{i} B_{i}^{*}, B_{i} \in A_{2}\left(\pi_{2}\right)$, an approximate identity of $A^{\beta} \otimes$ $B\left(H_{\pi_{2}}\right)$. We have

$$
A_{1} \odot B_{j}=\left[A_{i}\left[\begin{array}{ccc}
b_{r s}^{j} & & 0 \\
& \ddots & \\
0 & & b_{r s}^{j}
\end{array}\right]\right], \quad B_{j}=\left[b_{r s}^{j}\right] .
$$

Hence,

$$
\left[A_{i} \odot B_{j}\right]\left[A_{i} \odot B_{j}\right]^{*}=\left[A_{i}\left[\begin{array}{ccc}
\sum_{p} b_{r p}^{j} b_{s p}^{j^{*}} & & 0 \\
& \ddots & \\
0 & & \sum_{p} b_{r p}^{j} b_{s p}^{j^{*}}
\end{array}\right] A_{i}^{*}\right] .
$$

Since

$$
e_{2}^{\lambda_{2}} \underset{\lambda_{2}}{\stackrel{(s)}{\longrightarrow}} I
$$

we have

$$
\sum_{j=1}^{m_{\lambda_{2}}}\left[A_{i} \odot B_{j}\right]\left[A_{i} \otimes B_{j}\right]^{*} \underset{\lambda_{2}}{\rightarrow}\left[\begin{array}{ccccc}
A_{i} & A_{i}^{*} & & 0 & \\
& & \ddots & & \\
& 0 & & A_{i} & A_{i}^{*}
\end{array}\right] .
$$

Since $e_{1}^{\lambda_{1}} \stackrel{(s)}{\rightarrow} I$ we then obtain that

$$
\left.\overline{\left[A_{2}^{\beta}\left(\pi_{1}\right) \odot A_{2}^{\beta}\left(\pi_{2}\right)\right]\left[A_{2}^{\beta}\left(\pi_{1}\right) \odot A_{2}^{\beta}\left(\pi_{2}\right)\right.}\right]^{*}=A^{\beta} \otimes B\left(H_{\pi_{1} \otimes \pi_{2}}\right) .
$$

From this equality it follows that

$$
A_{2}^{\beta}\left(\pi_{1} \otimes \pi_{2}\right)=A^{\beta} \otimes B\left(H_{\pi_{1} \otimes \pi_{2}}\right) .
$$

Since $A_{2}^{\beta}\left(\pi_{1} \otimes \pi_{2}\right)=\sum_{j}^{\oplus} A_{2}^{\beta}\left(\pi^{j}\right)$ where $\pi_{1} \otimes \pi_{2}=\sum_{i}^{\oplus} \pi^{j}$ it follows that $A_{2}^{\beta}\left(\pi^{j}\right) \neq 0$ for every $j$ and the condition (b) is proven.

By the faithfulness of $\beta, P$ separates the points of $K$. We can now apply [5, Theorem 27.39(iii)] to obtain $P=\Pi(K)$ and the proof of Proposition 5.2 is complete.

5.3 Proposition. Let $(A, K, \beta)$ be a $C^{*}$-dynamical system with $K$ compact and $\beta$ faithful. Assume that there is a minimal action $(G, \alpha)$ on $A$ such that $\alpha_{G} \operatorname{Aut}_{\beta}(A)$. Then the following conditions are equivalent:

(1) $A \times_{\beta} K$ is simple.

(2) $A^{\beta}$ is simple.

Proof. By Theorem A (1) $\rightarrow(2)$. Assume that (2) holds. It can be easily checked that a minimal action is topologically transitive. Therefore by Proposition $5.2 A_{1}^{\beta}(\pi) \neq(0)$ for every $\pi \in \widehat{K}$. Hence, taking into account Theorem 
A, in order to prove that (1) holds, we have to prove that for every $\pi \in \widehat{K}$ the algebra $A \otimes B\left(H_{\pi}\right)^{\alpha \otimes a d} \pi$ is simple. Since $A_{1}^{\beta}(\pi) \neq(0)$ by $\S 2(8)$ it follows that $A_{2}^{\beta}(\pi) \neq(0)$. It is easy to see that $A_{2}^{\beta}(\pi) A_{2}^{\beta}(\pi)^{*}=A^{\beta} \otimes B\left(H_{\pi}\right)$ which is a simple $C^{*}$-algebra by assumption. It can be immediately verified that $A_{2}^{\beta}(\pi)$ is an $A_{2}^{\beta}(\pi) A_{2}^{\beta}(\pi)^{*}-A_{2}^{\beta}(\pi)^{*} A_{2}^{\beta}(\pi)$ imprimitivity bimodule (in the sense of [11]). By [12, Theorem 3.1] $A_{2}^{\beta}(\pi)^{*} A_{2}^{\beta}(\pi)$ is simple. We shall prove that $\overline{A_{2}^{\beta}(\pi)^{*} A_{2}^{\beta}(\pi)}=A \otimes B\left(H_{\pi}\right)^{\beta \otimes \text { ad } \pi}$ so that $A \otimes B\left(H_{\pi}\right)^{\beta \otimes \mathrm{ad} \pi}$ will be proved to be simple.

By virtue of Remark 3.2 there exists an approximate identity $\left(e_{\lambda}\right)$ of $A_{2}^{\beta}(\pi)^{*} A_{2}^{\beta}(\pi)$ of the form $e_{\lambda}=\sum_{i=1}^{n_{\lambda}} a_{i}^{*} a_{i}$ where $a_{i} \in A_{2}^{\beta}(\pi)$. Denote $e_{\lambda}=\left[e_{i j}^{\lambda}\right]$ with $e_{i j}^{\lambda} \in A$. Then $\sup _{\lambda \in \Lambda} e_{\lambda}=e$ where $e=\left[e_{i j}\right]$ is the unit of $\overline{A_{2}^{\beta}(\pi)^{*} A_{2}^{\beta}(\pi)^{\sigma}}$ (where $\overline{A_{2}^{\beta}(\pi)^{*} A_{2}^{\beta}(\pi)^{\sigma}}$ is the ultraweak closure of $A_{2}^{\beta}(\pi)^{*} A_{2}^{\beta}(\pi)$ ). By Remark $3.1 \tilde{\alpha}_{g}(e)=e$ for every $g \in G$. Therefore, $\alpha_{g}\left(e_{i j}\right)=e_{i j}, g \in G, i \leq i, j \leq$ $\operatorname{dim} \pi$. In particular, $e_{i i} \quad(1 \leq i \leq \operatorname{dim} \pi)$ is a lower semicontinuous, positive, element in $A^{\prime \prime}$ (that is $e_{i i}$ is the strong limit of an increasing net of positive elements of $A$ ). By [1, Proposition 4.1] $e_{i i}$ are all scalar multiples of the identity, $e_{i i}=\lambda_{i i} 1$. We shall now prove that all $e_{i j}, 1 \leq i, j \leq \operatorname{dim} \pi$ are scalar multiples of the identity. Denote by $H$ the Hilbert space of the universal representation of $A$ and let $\xi \in H$ be arbitrary.

Notice that $H \otimes H_{\pi} \simeq \bigoplus_{i=1}^{\operatorname{dim} \pi} H_{i}, H_{i}=H$ for every $1 \leq i \leq \operatorname{dim} \pi$. Consider the following element $\xi_{i j}$ of $H \otimes H_{\pi}$ :

$$
\xi_{i j}=0 \oplus \cdots \oplus \xi_{i} \oplus 0 \oplus \cdots \oplus \xi_{j} \oplus 0 \oplus \cdots \oplus 0 .
$$

Taking into account that $\sup e_{\lambda}=e$ and that $e_{i i}=\lambda_{i i}, \lambda_{i i} \in R_{+}, i \leq i \leq$ $\operatorname{dim} \pi$, we have

$$
\begin{aligned}
\left\langle e \xi_{i j}, \xi_{i j}\right\rangle & =\left\langle e_{i i} \xi, \xi\right\rangle+\left\langle e_{i j} \xi, \xi\right\rangle+\left\langle e_{i j} \xi, \xi\right\rangle+\left\langle e_{j j} \xi, \xi\right\rangle \\
& =\left\langle\left(e_{i j}+e_{i j}^{*}\right) \xi, \gamma\right\rangle+\left(\lambda_{i i}+\lambda_{j j}\right)\|\xi\|^{2} \\
& =\sup _{\lambda}\left\langle\left(e_{i j}^{\lambda}+e_{i j}^{\lambda *}\right) \xi, \xi\right\rangle+\left(\lambda_{i i}+\lambda_{j j}\right)\|\xi\|^{2} .
\end{aligned}
$$

Therefore $e_{i j}+e_{i j}^{*}$ is a selfadjoint lower semicontinuous element in $A^{\prime \prime}$. Again applying [1, Proposition 4.1] we infer that $e_{i j}+e_{i j}^{*}$ is a scalar multiple of the identity. Similarly considering

$$
\bar{\xi}_{i j}=0 \oplus 0 \oplus \cdots \oplus 0 \oplus(-\xi) \oplus 0 \oplus \cdots \oplus \xi \oplus 0 \oplus \cdots \oplus 0
$$

we infer that $e_{i j}-e_{i j}^{*}$ is a scalar multiple of the identity. Therefore $e_{i j}=\lambda_{i j} I$, $\lambda_{i j} \in C$. Hence all the entries of $e=\left(e_{i j}\right)$ are scalar multiples of the identity. Since $e \in\left[A \otimes B\left(H_{\pi}\right)^{\beta \otimes \mathrm{ad} \pi}\right]^{\prime \prime} \subset A^{\prime \prime} \otimes B\left(H_{\pi}\right)^{\beta^{\prime \prime} \otimes \mathrm{ad} \pi}$ we obtain $e=I$. We then proved that $\overline{A_{2}^{\beta}(\pi)^{*} A_{2}^{\beta}(\pi)}=A \otimes B\left(H_{\pi}\right)^{\beta \otimes \text { ad } \pi}$, so $A \otimes B\left(H_{\pi}\right)^{\beta \otimes \mathrm{ad} \pi}$ is simple. By Theorem A we have that $A \times_{\beta} K$ is simple.

We will now prove a result concerning the primeness of the crossed product $A \times{ }_{\beta} K$.

5.4 Proposition. Let $(A, K, \beta)$ be a $C^{*}$-dynamical system with $K$ compact and $\beta$ faithful. Assume that there is a strongly topologically transitive group action $(G, \alpha)$ on $A$ such that $\alpha_{G} \subset \operatorname{Aut}_{\beta}(A)$. Then the following conditions are equivalent: 
(1) $A \times_{\beta} K$ is prime,

(2) $A^{\beta}$ is prime.

Proof. By Theorem A (1) $\rightarrow(2)$. Assume that (2) holds. By [2, Corollary 2.2] $A_{1}^{\beta}(\pi) \neq(0)$ for every $\pi \in \widehat{K}$ and the condition (2)(b) of Theorem $\mathrm{A}$ is satisfied. Hence in order to prove that (1) holds it is enough to prove that the fixed point algebras $A \otimes B\left(H_{\pi}\right)^{\beta \otimes \text { ad } \pi}$ are simple for all $\pi \in \widehat{K}$. Let $\pi \in \widehat{K}$. Then the ideal $A_{2}^{\beta}(\pi)^{*} A_{2}^{\beta}(\pi)$ of $A \otimes B\left(H_{\pi}\right)^{\beta \otimes a d} \pi$ is a prime $C^{*}$-algebra. Indeed, $A_{2}^{\beta}(\pi)$ is an $A_{2}^{\beta}(\pi)^{*} A_{2}^{\beta}(\pi)-A_{2}^{\beta}(\pi) a_{2}^{\beta}(\pi)^{*}$-imprimitivity bimodule. Since $A^{\beta}$ is prime by assumption, it follows that $A_{2}^{\beta}(\pi) \otimes B\left(H_{\pi}\right)$ is prime, so its ideal $\overline{A_{2}^{\beta}(\pi) A_{2}^{\beta}(\pi)^{*}}$ is prime. By [13, Theorem 3.1] it follows that $\overline{A_{2}^{\beta}(\pi)^{*} A_{2}^{\beta}(\pi)}$ is a prime $C^{*}$-algebra. We will now prove that $A_{2}^{\beta}(\pi)^{*} A_{2}^{\beta}(\pi)$ is an essential ideal of $A \otimes B\left(H_{\pi}\right)^{\beta \otimes \text { ad } \pi}$ i.e. that $C \cdot A_{2}^{\beta}(\pi)^{*} A_{2}^{\beta}(\pi) \neq(0)$ for every $C \in A \otimes B\left(H_{\pi}\right)^{\beta \otimes a d} \pi$, $C \neq 0$. Assume by contradiction that there is a $C \in A \otimes B\left(H_{\pi}\right)^{\beta \otimes \mathrm{ad} \pi}$ such that $C A_{2}^{\beta}(\pi)^{*} A_{2}^{\beta}(\pi)=(0)$. Then $A_{2}^{\beta}(\pi) C=(0)$.

Therefore $\left[P_{\pi, i, j}^{\beta}(a)\right]\left[C_{i j}\right]=0$ for every $a \in A$, where $C=\left[C_{i j}\right]$. Let $a \in A$ be such that $P_{\pi}^{\beta}(a) \neq 0$. Then by $\S 2$, (8) $P_{\pi, i, i}^{\beta}(a) \neq 0$ for some $i$. We can assume that $P_{\pi, 1,1}^{\beta}(a) \neq 0$. Denoting $P_{\pi, 1,1}^{\beta}(a)=a_{11}$ we have by $\S 2,(3)(\mathrm{b})$ :

$$
P_{\pi, i, j}^{\beta}\left(a_{11}\right)=\delta_{i 1} P_{\pi, 1, j}^{\beta}(a)
$$

In particular $\left[P_{\pi, i, j}^{\beta}\left(a_{11}\right)\right] \cdot\left[C_{i j}\right]=0$. Therefore

$$
\sum_{i=1}^{\operatorname{dim} \pi} P_{\pi, 1, j}^{\beta}\left(a_{11}\right) C_{i j}=0 .
$$

Since $\alpha$ commutes with $\beta$ we also have

$$
\sum \alpha_{g} P_{\pi, 1, j}^{\beta}\left(a_{11}\right) C_{i j}=\sum P_{\pi, 1, j}^{\beta}\left(\alpha_{g}\left(a_{11}\right)\right) C_{i j}=0,
$$

for every $g \in G$ and $1 \leq i \leq \operatorname{dim} \pi$.

But it is straightforward to check that $P_{\pi, 1, j}^{\beta}(a)$ are linearly independent so that if $C \neq 0$ there is an $i$ such that $\sum P_{\pi, 1, j}^{\beta}\left(a_{11}\right) \otimes C_{i j} \neq 0$. This, together with (2) contradicts the strong topological transitivity of $\alpha$. Therefore, we have proved that the ideal $A_{2}^{\beta}(\pi)^{*} A_{2}^{\beta}(\pi)$ is prime and essential. Then the algebra $A \otimes B\left(H_{\pi}\right)^{\beta \otimes a d} \pi$ itself is prime, and the proof is complete.

The above proof does not seem to carry over to the case of topologically transitive actions. So the following problem presents itself: Can Proposition 5.2 be stated for topologically transitive actions $(G, \alpha)$ instead of strong topologically transitive ones?

Another problem that could shed an interesting light on the relationship between the simplicity or primeness of the crossed product and the ergodicity of the commutant of the action is the following:

Does the simplicity (primeness) of the crossed product $A \times{ }_{\beta} K$ imply the minimality (topological transitivity) of the commutant of $\beta_{K}$ in $\operatorname{Aut}(A)$ ? 


\section{REFERENCES}

1. D. Avitzour, Noncommutative topological dynamics. I, Trans. Amer. Math. Soc. 282 (1984), 109-119.

2. O. Bratteli, G. A. Elliott and D. W. Robinson, Strong topological transitivity anc' $C^{*}$ dynamical systems, J. Math. Soc. Japan 37 (1985), 115-133.

3. O. Bratteli, Derivations, dissipations and group actions on $C^{*}$-algebras, Lecture Notes in Math., vol. 1229, Springer-Verlag, 1986.

4. L. G. Brown, Stable isomorphism of hereditary subalgebras of $C^{*}$-algebras, Pacific J. Math. 71 (1977), 335-349.

5. E. Hewitt and K. A. Ross, Abstract harmonic analysis, vol. II, Springer-Verlag, 1970.

6. A. A. Kirillov, Elements of the theory of representations, Springer-Verlag, Berlin, 1976.

7. M. B. Landstad, Operator algebras and compact groups in operator algebras and group representation, Pitman, 1984.

8. R. Longo and C. Peligrad, Noncommutative topological dynamics and compact actions on $C^{*}$-algebras, J. Funct. Anal. 58 (1984), 157-175.

9. G. K. Pedersen, $C^{*}$-algebras and their automorphism groups, Academic Press, New York, 1979.

10. C. Peligrad, Locally compact group actions on $C^{*}$-algebras and compact subgroups, J. Funct. Anal. 76 (1988), 126-139.

11. N. C. Phillips, $K$-theoretic freeness of actions of finite groups on $C^{*}$-algebras, Thesis, Berkeley, 1984-1985.

12. M. A. Rieffel, Induced representations of $C^{*}$-algebras, Adv. in Math. 13 (1974), 176-251.

13. _ Unitary representations of group extensions, an algebraic approach to the theory of Mackey and Blattner, Studies in Analysis, Adv. in Math. Suppl. Series 4 (1979), 364-386.

14. M. Takesaki, Fourier analysis of compact automorphism groups (an application of Tannaka duality theorem), Colloq. Internat. du CNRS, no. 274, 1979.

Department of Mathematical Sciences, University of Cincinnati, Cincinnati, Ohio 45221-0025 